\title{
PAPER
}

\section{Significance of white matter high intensity lesions as a predictor of stroke from arteriolosclerosis}

\section{H Yamauchi, H Fukuda, C Oyanagi}

See end of article for authors' affiliations

.....................

Correspondence to: Dr Hiroshi Yamauchi Research Institute, Shiga Medical Centre, 5-4-30 Moriyama, Moriyama city, Shiga 524-8524, Japan; yamauchi@

shigamed.moriyama.shiga.jp

Received 14 May 2001

In revised form

30 August 2001

Accepted

27 September 2001

\begin{abstract}
Objectives: To determine whether the extent of white matter high intensity lesions (WML) on magnetic resonance imaging (MRI) is an independent predictor of risk for stroke from arteriolosclerosis, and whether serial evaluation of WML can be used to identify patients who are at risk of strokes.

Methods: Prospective follow up with serial MRI scans was done in 89 patients who were either diagnosed as having symptomatic lacunar infarcts or were stroke-free, neurologically normal individuals with headache or dizziness. None had significant stenosis of major cerebral arteries or atrial fibrillation. Multivariable analysis with the Cox proportional hazards model was used to test the predictive value for subsequent stroke of risk factor status at entry and during follow up, lacunar infarction, and the extent of WML (scored from 0 to 16 ) on the baseline scans.

Results: During follow up (mean (SD), 51 (19) months), seven strokes occurred (five lacunar infarcts and two haemorrhages): four in nine patients with severe WML (score 9-16), and three in 40 patients with mild WML (score 1-8) (log-rank test; $p<0.005$ ). None of 40 patients without WML experienced stroke. The extent of WML was an independent predictor of subsequent stroke (relative risk for a 1 point score increase, $1.60 ; 95 \%$ confidence interval, 1.02 to $2.54 ; p<0.05)$. In three strokes among 80 patients without severe WML, two occurred in four patients with an increase in WML score during follow up, and one occurred in the other 76 patients without an increased score $(p<0.0001)$.

Conclusions: Severe WML at baseline is an independent predictor of risk for stroke from arteriolosclerosis, while progression of WML during follow up may be associated with subsequent stroke in patients with initially mild WML.
\end{abstract}

W hite matter high intensity lesions (WML) on T2 weighted magnetic resonance images are often detected in elderly people. ${ }^{12}$ When located in the deep and subcortical white matter, WML may reflect ischaemic damage and correspond not only to focal rarefaction of myelin but also to loss of fibres and sometimes to lacunar infarcts, according to the histopathological characteristics. ${ }^{3}$ Arteriolosclerosis appears to be the most important causative factor in the development of such lesions, and the extent of WML is thought to reflect the extent of brain arteriolosclerosis. ${ }^{4}$ Thus a predictive value of the extent of WML for strokes from arteriolosclerosis (lacunar infarcts or haemorrhages) is biologically plausible. However, it is unclear whether the extent of WML allows one to define subgroups of patients at high risk for subsequent stroke beyond what can be expected from simple clinical examination. ${ }^{15}$

There are few longitudinal data on stroke occurrence in the subgroup of patients categorised by the extent of WML on magnetic resonance images, ${ }^{67}$ although several studies using computed tomography (CT) have investigated the relation between leucoaraiosis and stroke risk..$^{8-12}$ The results of CT studies may not be extendable to magnetic resonance imaging, because the lesions visualised by these two techniques cannot be completely superimposed for number, site, and extension. ' Some CT studies have shown that extensive leucoaraiosis predicts future stroke, ${ }^{8-11}$ suggesting that WML of the most severe degrees may indicate increased stroke risk. However, other investigators ${ }^{12}$ have failed to confirm this finding, and it is unclear whether leucoaraiosis on CT is a predictor of risk for subsequent strokes from arteriolosclerosis. ${ }^{8}$ On the basis of the hypothesis that extensive WML is an indicator of diffuse arteriolosclerosis leading to stroke, it is highly plausible that WML has predictive value for stroke in patients with lacunar infarction. However, even in patients with lacunar infarcts the results of studies investigating the predictive value of WML or leucoaraiosis for stroke are conflicting. ${ }^{6}{ }^{12}$

The inconsistency among previous studies may reflect differences in risk factor status during follow up, as well as the status of the patients at entry. Few studies have analysed the strength and independence of the predictive value of WML for stroke after controlling for the risk factor status during follow up. Furthermore, longitudinal data on the relation between progression of WML and subsequent stroke should resolve the controversy about the association between WML and stroke risk. Population based studies on the rate of WML progression have shown an asymptomatic increase in the number and extent of WML in relation to hypertension. ${ }^{13}{ }^{14}$ However, little is known about the relation between progression of WML and the risk of subsequent stroke.

In this study, we selected 89 patients who either had symptomatic lacunar infarcts or or were stroke-free, neurologically normal individuals with headache or dizziness. None had non-lacunar infarction, significant stenosis of major cerebral arteries, or atrial fibrillation. We followed up them with serial magnetic resonance imaging (MRI) scans until the occurrence of stroke or death. The relation between the subsequent risk of stroke and the extent of WML was analysed after adjustment for several clinical variables including vascular risk factors in the controls during follow up. The relation between the increase in WML and the risk of subsequent stroke was also analysed. Our objectives were to determine whether the extent of WML at baseline was an independent predictor of risk for subsequent stroke on the basis of arteriolosclerosis, and whether serial evaluation of the extent of WML could be used to identify patients with initially mild WML who were at high risk of subsequent stroke. 


\section{METHODS \\ Patients}

We studied 89 consecutive outpatients aged 43 to 88 years (mean (SD) age, 66 (9) years), who were either diagnosed as having symptomatic lacunar infarcts or were stroke-free, neurologically normal individuals with headache or dizziness. There were 38 men and 51 women. All subjects were selected prospectively from patients with neurological symptoms who visited the outpatient department of neurology at Saiseikai Noe Hospital on Thursdays between April 1993 and March 1998, and underwent MRI to diagnose or rule out central nervous system diseases.

Inclusion criteria were as follows:

- patients with a history of lacunar stroke, a clinical presentation consistent with one of the lacunar syndromes described by Fisher, ${ }^{15}$ and MRI evidence of lacunar infarcts that appeared to be responsible for their symptoms;

- patients who underwent MRI because of headache or dizziness and had normal neurological findings and no specific neurological diseases other than tension-type headache, irrespective of MRI evidence of infarcts or any degree of WML.

Exclusion criteria were as follows:

- cortical infarct on MRI;

- significant stenosis of the cervical or intracranial arteries on magnetic resonance angiography;

- complications of other neurological or psychiatric disorders, including alcohol abuse and depression;

- patients who did not need follow up because they had normal MRI findings, no symptoms after initial MRI examination, and no vascular risk factors.

In all, 144 patients satisfied the inclusion criteria. Among 43 patients with a history of lacunar stroke, two with stenosis of the middle cerebral artery were excluded. Among 101 strokefree patients, two with depression were excluded. Fifty one patients were also excluded because they had normal MRI findings, were symptom-free, and had no vascular risk factors. None had cortical infarction on MRI or atrial fibrillation on ECG. In patients with ischaemic heart disease, none showed sources of embolism on echocardiography.

Forty two of the 89 patients had lacunar infarcts on MRI. A lacunar infarct was identified as an increased signal intensity on T2 weighted images, with clearly delineated decreased signal intensity on $\mathrm{Tl}$ weighted images. No patient had lesions with a diameter of more than $1.5 \mathrm{~cm}$. Twenty one of these 42 patients had multiple lacunar infarcts, most of which were located in the basal ganglia and were not considered compatible with the clinical symptoms and signs. There were 41 patients with symptomatic lacunar stroke and one with an asymptomatic lacunar infarct in the putamen. These comprised 27 men and 15 women, aged 50 to 82 years (mean (SD), 68 (8) years). The other 47 patients without lacunar infarcts comprised 11 men and 36 women aged 43 to 88 years (mean 65 (10) years).

Patients were followed up at Saiseikai Noe Hospital. Treatment of risk factors and the use of drugs were left to individual clinical judgment. In principle, however, antiplatelet agents (aspirin or ticlopidine $\mathrm{HCl}$ ) were given to patients with lacunar stroke if they did not have contraindication to this treatment or poor blood pressure control. Although the attending physicians were not blinded to the findings of the MRI studies, treatment did not differ markedly among the patients. All patients were examined at two month intervals or more often after the baseline MRI scans. At each visit, an interim history was obtained and a neurological examination was performed. In all patients, follow up MRI scans were repeated at intervals of 12 to 18 months.
The vascular risk factors evaluated were hypertension, diabetes mellitus, ischaemic heart disease, hypercholesterolaemia, and smoking; information on these was obtained from clinical history and laboratory findings at baseline and during follow up. Hypertension at entry was judged to be present when there was a systolic blood pressure of more than 160 $\mathrm{mm} \mathrm{Hg}$ or a diastolic blood pressure of more than $95 \mathrm{~mm} \mathrm{Hg}$ on repeated measurements, or when there was a history of treatment for hypertension. ${ }^{16}$ Blood pressure was measured at each visit. The mean values of blood pressure during follow up were judged as controlled (systolic pressure less than $140 \mathrm{~mm}$ $\mathrm{Hg}$ and diastolic pressure less than $90 \mathrm{~mm} \mathrm{Hg}$ ), mild hypertension (systolic pressure 140-159 mm Hg, or diastolic pressure 90-99 $\mathrm{mm} \mathrm{Hg}$ ), or moderate hypertension (systolic pressure more than $159 \mathrm{~mm} \mathrm{Hg}$ or diastolic pressure more than $99 \mathrm{~mm} \mathrm{Hg}){ }^{17}$

Diabetes mellitus at entry was judged to be present when the fasting blood glucose concentration was more than 140 $\mathrm{mg} / \mathrm{dl}$ or when there was a history of treatment for diabetes mellitus. The blood glucose level and glycated haemoglobin concentration (HbAlc value) were measured once a month in patients with diabetes mellitus and once a year or more often in those without. The blood glucose concentration during follow up was judged as controlled when the mean value of HbAlc was less than $7 \%$.

Ischaemic heart disease at entry was judged to be present when there was a history of angina pectoris or myocardial infarction.

Hypercholesterolaemia at entry was judged to be present when the serum total cholesterol was more than $6.2 \mathrm{mmol} / \mathrm{l}$ $(240 \mathrm{mg} / \mathrm{dl})$ or when there was a history of treatment. The cholesterol level was measured at least once a year. The cholesterol level during follow up was judged as controlled when the mean value of serum total cholesterol was less than 5.7 $\mathrm{mmol} / \mathrm{l}(220 \mathrm{mg} / \mathrm{dl})$.

End points were defined as the occurrence of stroke or death. In patients with stroke, MRI or CT was undertaken and compared with the initial studies to confirm the occurrence of stroke.

\section{MRI}

MRI was done with a Vectra unit (General Electric, Milwaukee, Wisconsin, USA) operating at a field strength of $0.5 \mathrm{~T}$. Tl weighted axial images were obtained with the use of a spin echo pulse sequence (repetition time, $440 \mathrm{~ms}$; echo time, $17 \mathrm{~ms}$ ). Axial proton weighted and T2 weighted images were also obtained with spin echo pulse sequences (repetition time, $3500 \mathrm{~ms}$; echo time, 40 and $90 \mathrm{~ms}$, respectively). The slice thickness was $7 \mathrm{~mm}$. Sections had intersectional gaps of 1.9 $\mathrm{mm}$. In all patients with symptomatic lacunar stroke, the baseline MRI scans for this study were performed at least one month after the ischaemic event.

\section{White matter high intensity lesions}

To grade WML, axial T2 weighted images were evaluated visually, and separately for each hemisphere. The presence, location, and degree of white matter lesions were assessed according to a recently described scale. ${ }^{18}$ This scale requires separate evaluation of the anterior and posterior regions of each hemisphere on three MRI slices: one through the choroid plexus of the posterior horns, one through the cella media, and one through the centrum semiovale. The first two slices were used to evaluate the anterior region (the region around the anterior horn of the lateral ventricles); the other two were used to evaluate the posterior region (the white matter around the posterior part of the body of the lateral ventricles and the posterior part of the centrum semiovale). For this study a modification was made in order to allow WML to be rated separately in each of the two regions on the three slices.

WML were identified as areas of increased signal intensity on T2 weighted images. Most WML were visualised as high 
Table 1 Characteristics of the patients

\begin{tabular}{|c|c|c|c|}
\hline \multirow[b]{2}{*}{ Characteristic } & \multicolumn{2}{|l|}{ WML } & \multirow[b]{2}{*}{$\mathrm{p}$ Value } \\
\hline & Present* & Absent & \\
\hline Number of patients & 49 & 40 & \\
\hline \multicolumn{4}{|l|}{ WML } \\
\hline Mild & 40 & & \\
\hline Severe & 9 & & \\
\hline Lacunar infarction $\ddagger$ & 30 & 12 & 0.0064 \\
\hline Multiple infarction & 18 & 3 & 0.0029 \\
\hline Age (years) & $68.4(8.3)$ & $63.4(9.8)$ & 0.011 \\
\hline \multicolumn{4}{|l|}{ Sex } \\
\hline Male/female & $25 / 24$ & $13 / 27$ & 0.13 \\
\hline Follow up time (months) & $51.1(21.2)$ & $51.9(17.3)$ & 0.86 \\
\hline \multicolumn{4}{|l|}{ Other medical illness } \\
\hline Hypertension & 22 & 6 & 0.0052 \\
\hline Diabetes mellitus & 13 & 3 & 0.041 \\
\hline Ischaemic heart disease & 6 & 2 & 0.41 \\
\hline Hypercholesterolaemia & 9 & 4 & 0.42 \\
\hline Smoking ever & 9 & 2 & 0.11 \\
\hline Current smoking & 6 & 1 & 0.19 \\
\hline Past smoking & 3 & 1 & 0.76 \\
\hline Antiplatelet treatment & 26 & 13 & 0.09 \\
\hline \multicolumn{4}{|c|}{$\begin{array}{l}\text { Values are mean }(S D) \text { or } n \text {. } \\
\text { *WML score }>0 \text {. } \\
\text { † } \chi^{2} \text { test or Student's } t \text { test, as appropriate. } \\
\ddagger \text { Symptomatic or asymptomatic lacunar infarcts on MRI. } \\
\text { MRI, magnetic resonance imaging; WML, white matter high intensity } \\
\text { lesions on T2 weighted MR images. }\end{array}$} \\
\hline
\end{tabular}

intensity areas on proton weighted images and as normointense areas or poorly delineated hypointense areas on $\mathrm{Tl}$ weighted images. Focal lesions were judged as present when the diameter was $2 \mathrm{~mm}$ or more. The severity of WML on T2 weighted images, including areas of infarct on Tl weighted images, was graded on a two point scale in each of the two regions on the three slices, according to their number and configuration. A score of 0 denoted no lesion or only a single lesion; a score of 1 denoted multiple focal lesions; a score of 2 denoted multiple confluent lesions scattered throughout the white matter. Total WML scores were calculated by summing the scores in each region in both hemispheres, with a maximum score of 16 and a minimum score of 0 . We also graded the overall severity of WML as a simple rating of none, mild (score 1-8), or severe (score 9-16).

The grading of WML was done by one investigator who was blinded to the clinical status of the patients. To investigate the change in WML grade, all initial and follow up scans (before the occurrence of stroke, if any) in each patient were read side by side, and the sequence of the scans was known to the reader. We chose this method because we thought it would allow optimal evaluation, even though independent assessment of the scans might have been preferable from the point of view of avoiding bias.

Before the study, observer reliability for grading the severity of WML was evaluated from a blind re-review of 50 MRI scans in patients with strokes. There was high intraobserver and interobserver reliability for the grading system used. The intraclass correlation coefficient for intraobserver reliability was 0.98 and for interobserver reliability, 0.94 .

\section{Statistical analysis}

We compared the clinical background between patients with WML and those without using Student's $t$ test or the $\chi^{2}$ test as appropriate. Stepwise regression analysis was used in all patients to test the independent predictive value of multiple variables at entry with respect to the WML score on the baseline scans. In this analysis the WML score was the dependent variable and the patient's age, sex, the presence of other medical illness (hypertension, diabetes mellitus, or ischaemic heart disease), smoking status, and the presence of single or multiple lacunar infarcts as the independent variables. We also compared the incidence of subsequent stroke among patients with severe WML, mild WML, and no WML, using the Mantel-Cox log-rank statistics and Kaplan-Meier survival curves. $^{19}$

Single variable or multivariable analysis with the Cox proportional hazards model was used to test the effect of multiple variables on the occurrence of stroke. ${ }^{20}$ The age, sex, presence of single lacunar infarct, presence of multiple lacunar infarcts, presence (at entry) or control status (during follow up) of other medical illness (hypertension, diabetes mellitus, ischaemic heart disease, hypercholesterolaemia), smoking status (current or past), and WML scores were considered covariates.

In patients without a severe degree of WML, stepwise regression analysis was used to test the independent predictive values of multiple variables with respect to the increase of WML score during follow up. The increased value of the WML score (before the occurrence of stroke, if any) was used as the dependent variable, and the age, sex, presence or control status of other medical illness, smoking status, presence of single or multiple lacunar infarcts, and WML scores at baseline were used as the independent variables.

In patients with an increase in WML score, the mean values of blood pressure, $\mathrm{HbAlc}$, and total cholesterol during follow up until the detection of an increase in WML were also calculated and used for determining control status for this analysis. We also compared the incidence of subsequent stroke between patients with and without an increase in WML score using the Mantel-Cox log-rank statistics and Kaplan-Meier survival curves.

Significance was established at $\mathrm{p}<0.05$. Two tailed tests were used in all analyses. Statistical analyses were done using Statview for Macintosh (SAS Institute Inc, 1998).

\section{RESULTS}

Table 1 shows the clinical characteristics of patients with and without WML. Of the 89 patients, 49 had WML on the baseline

\begin{tabular}{|c|c|c|c|c|}
\hline Variable & Coefficient & SEM & $t$ Value & $\mathrm{p}$ Value \\
\hline \multicolumn{5}{|l|}{ Model 1} \\
\hline Age (years) & 0.12 & 0.037 & 3.12 & 0.0025 \\
\hline Multiple lacunar infarcts (no $=0$, yes $=1$ ) & 3.35 & 0.83 & 4.05 & 0.0001 \\
\hline Diabetes mellitus (no $=0$, yes $=1$ ) & 3.55 & 0.89 & 4.00 & 0.0001 \\
\hline Current smoking (no $=0$, yes $=1)$ & 3.86 & 1.31 & 2.95 & 0.0042 \\
\hline \multicolumn{5}{|l|}{ Model 2} \\
\hline Age (years) & 0.13 & 0.039 & 3.45 & 0.0009 \\
\hline Hypertension (no $=0$, yes $=1$ ) & 2.11 & 0.79 & 2.67 & 0.009 \\
\hline Diabetes mellitus $(\mathrm{no}=0$, yes $=1$ ) & 3.36 & 0.96 & 3.51 & 0.0007 \\
\hline Current smoking (no $=0$, yes $=1$ ) & 5.36 & 1.34 & 4.00 & 0.0001 \\
\hline
\end{tabular}




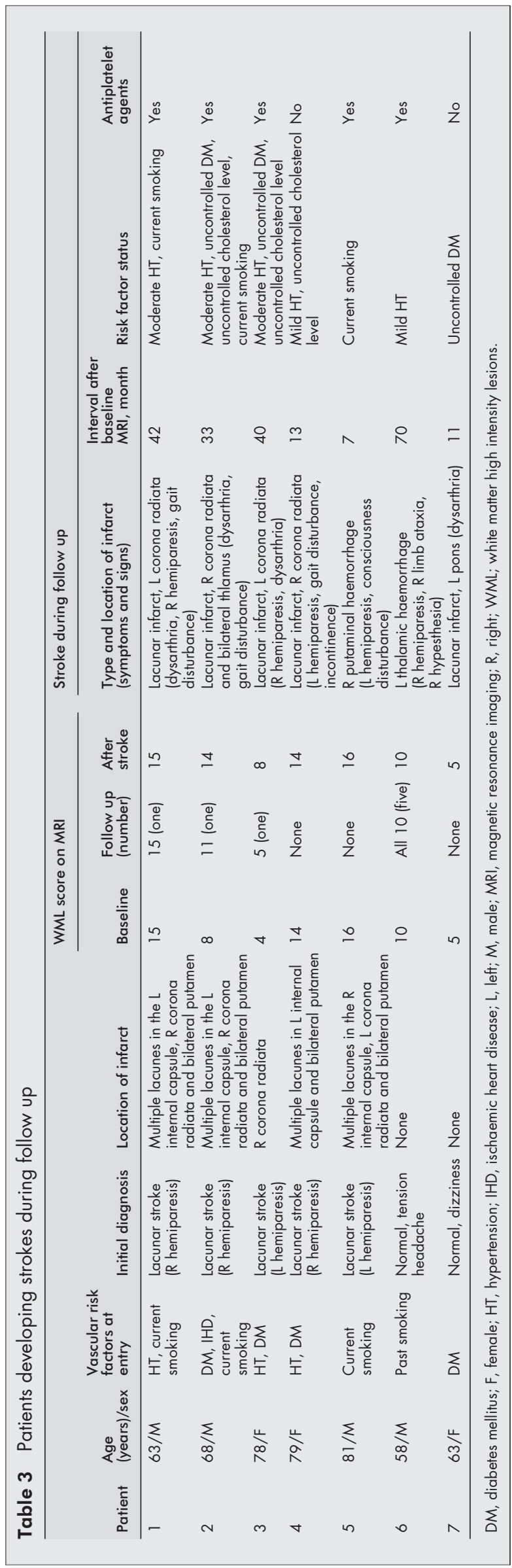

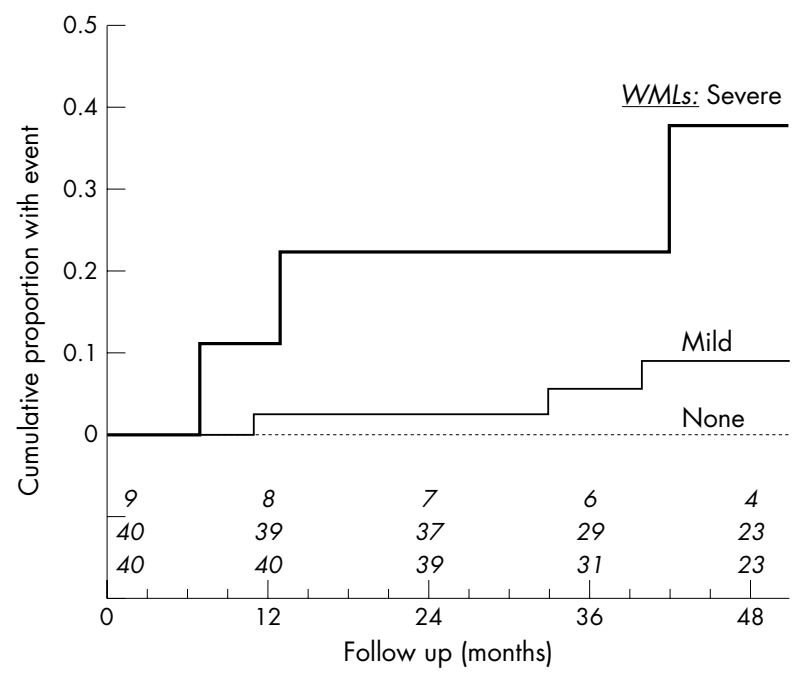

Figure 1 Kaplan-Meier cumulative failure curves for strokes in patients with no, mild, and severe white matter high intensity lesions (WML) on T2 weighted magnetic resonance images. All strokes occurred in the 49 patients with WML. The incidence of stroke was significantly higher in patients with severe WML (score 9-16) than in patients with mild WML (score 1-8) (log-rank test, $p<0.005)$. The number of patients who remained event-free and available for follow up evaluation during each 12 month interval is shown at the bottom of the graph. Top row, patients with severe WML; middle row, patients with mild WML; bottom row, patients without WML.

MRI scan-mild in 40 and severe in nine. A score of 1 was found in two patients, 2 in 10, 3 in three, 4 in eight, 5 in five, 6 in seven, 8 in five, 10 in two, 11 in two, 13 in one, 14 in one, 15 in one, and 16 in two. The distribution of age, lacunar infarct, multiple lacunar infarct, hypertension, and diabetes mellitus was significantly different between patients with WML and those without.

When demographic variables and vascular risk factors at entry were entered into a stepwise regression analysis, the patient's age, the presence of multiple lacunar infarcts, the presence of diabetes mellitus, and current smoking accounted for a significant proportion of the variances of the WML score (adjusted $R^{2}=0.42 ; \mathrm{p}<0.0001$ ); these four variables were independently correlated with the WML score (table 2, model 1). When the presence of lacunar infarction (single or multiple) was excluded as an independent variable, hypertension became a significant predictor (adjusted $R^{2}=0.36$; $\mathrm{p}<0.0001$ ) (table 2, model 2).

Follow up time in the 89 patients ranged from seven to 83 months (mean (SD), 51 (19) months). Antihypertensive agents were given to all patients with hypertension-calcium channel blockers in 20, angiotensin converting enzyme inhibitors in four, and both drugs in four. Twenty nine of the 42 patients with lacunar infarcts and 10 of the 47 without were treated with antiplatelet agents. In patients without lacunar infarcts, antiplatelet agents were given because of ischaemic heart disease, complications of diabetes mellitus, or severe WML. Two patients without lacunar infarcts dropped out of the study after being followed for 23 and 25 months, respectively, because they moved home. Their WML scores were 0 and 6 at entry and were unchanged on the follow up scans. While mean blood pressure during follow up was judged to be controlled in 54 patients, there was mild hypertension in 29 and moderate hypertension in six. The blood glucose level during follow up was considered uncontrolled in seven patients (all having poorly controlled diabetes mellitus). Blood cholesterol during follow up was considered uncontrolled in 21 patients. 
Table 4 Single variable and multivariable analyses of risk factors for the end point of subsequent stroke

\begin{tabular}{|c|c|c|c|c|}
\hline \multirow[b]{2}{*}{ Variable } & \multicolumn{2}{|l|}{ Single variable analysis } & \multicolumn{2}{|l|}{ Multivariable analysis } \\
\hline & $\begin{array}{l}\text { Hazard ratio ( } 95 \% \\
\text { confidence interval) }\end{array}$ & $\mathrm{p}$ Value & $\begin{array}{l}\text { Hazard ratio ( } 95 \% \\
\text { confidence interval) }\end{array}$ & $p$ Value \\
\hline WML score (point) at baseline scan & $1.39(1.18$ to 1.63$)$ & $<0.0001$ & $1.60(1.02$ to 2.54$)$ & 0.044 \\
\hline Uncontrolled diabetes mellitus (no=0, yes $=1$ ) & $9.74(2.17$ to 43.74$)$ & 0.030 & $68.36(1.33$ to 3513.25$)$ & 0.036 \\
\hline Age (years) & $1.07(0.98$ to 1.17$)$ & 0.14 & $1.083(0.92$ to 1.28$)$ & 0.35 \\
\hline Sex $($ male $=0$, female $=1)$ & & & $0.84(0.066$ to 10.68$)$ & 0.89 \\
\hline Moderate hypertension (no $=0$, yes $=1$ ) & 20.15 (3.86 to 105.29$)$ & 0.0004 & $0.73(0.034$ to 15.99$)$ & 0.84 \\
\hline Current smoking (no=0, yes $=1)$ & $9.56(2.13$ to 42.81$)$ & 0.0032 & $2.63(0.080$ to 86.54$)$ & 0.59 \\
\hline Multiple lacunar infarcts (no=0, yes $=1$ ) & $5.81(1.29$ to 26.25$)$ & 0.022 & $0.35(0.010$ to 12.26$)$ & 0.56 \\
\hline Antiplatelet agent (no $=0$, yes $=1$ ) & $7.26(0.87$ to 60.45$)$ & 0.067 & $11.33(0.26$ to 498.77$)$ & 0.21 \\
\hline
\end{tabular}

All variables with $p<0.20$ were listed in univariate analysis.

WML, white matter high intensity lesions on T2 weighted magnetic resonance images.

\section{Extent of WML at baseline and subsequent stroke}

During follow up, seven strokes occurred (table 3), all in the 49 patients with WML. No stroke other than lacunar infarction or cerebral haemorrhage occurred in the 89 patients. Five strokes were lacunar infarctions, including four supratentorial and one brain stem infarct, and two were haemorrhages, including one putaminal and one thalamic haemorrhage. Three strokes occurred in patients with mild degrees of WML (WML scores 4,5 , and 8), and four in patients with severe WML (WML scores 10, 14, 15, and 16). Death occurred in one patient with WML and in three patients without $\left(\chi^{2}\right.$ test, $\left.\mathrm{p}=0.47\right)$. The Kaplan-Meier cumulative failure curve is shown in fig 1. The incidence of stroke was significantly higher in the nine patients with severe WML than in the 40 patients with mild WML (log-rank test, $\mathrm{p}<0.005$ ).

In single variable analysis using the Cox proportional hazards model, the extent of WML and the presence of multiple lacunar infarcts at baseline scans and the presence of moderate hypertension, uncontrolled diabetes mellitus, and smoking during follow up were significant predictors of subsequent stroke (table 4). None of the vascular risk factors at entry was a significant predictor. When seven variables with probability values of $<0.20$ in univariate analysis were entered into the multivariate model along with sex, only the extent of WML at entry and the presence of uncontrolled diabetes mellitus during follow up were significant independent predictors of subsequent stroke; the relative risk for a 1 point increase in WML score was 1.60 (95\% confidence interval, 1.02 to $2.54 ; \mathrm{p}<0.05$ ) (table 4 ). When only the patients with lacunar infarcts were analysed, the extent of WML became the sole significant independent predictor of subsequent stroke.

\section{Progression of WML during follow up and subsequent stroke}

All but three patients with early occurrence of subsequent stroke underwent follow up MRI scans at least once. Asymptomatic progression of the WML (an increase in WML scores) was found on MRI before the occurrence of stroke in two patients with recurrent stroke; at the start of follow up, WML scores were 8 to 11 and 4 to 5 , respectively (fig 2, table 3 ). No progression of WML occurred in the other four patients with

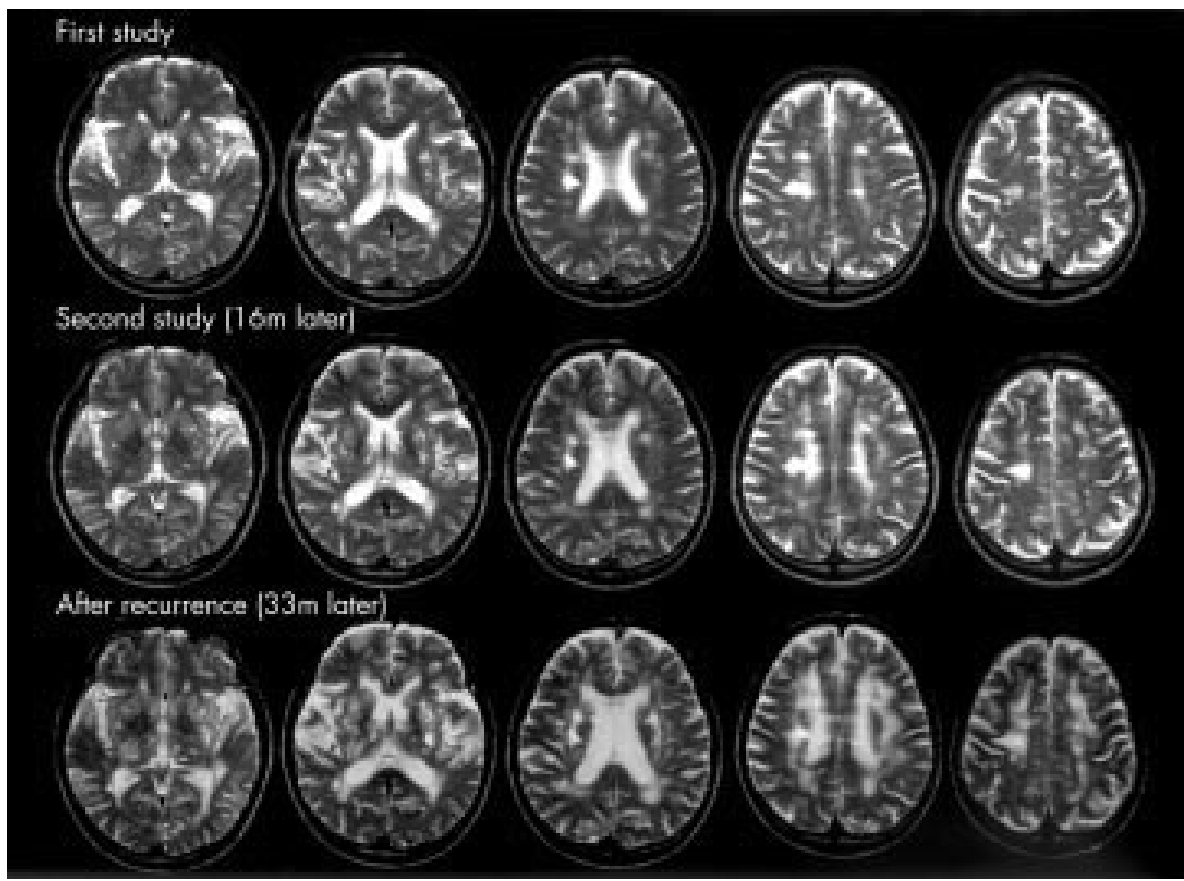

Figure 2 Example of progression of white matter high intensity lesions (WML) before occurrence of stroke in a 68 year old man with lacunar infarcts (patient 2 from table 3). The first magnetic resonance imaging (MRI) study (top row) shows multiple lacunar infarcts in the bilateral basal ganglia and WML (score = 8). The second study 16 months later (middle row) shows an asymptomatic increase in the number and extent of WML (score $=11$ ) in the bilateral hemispheres. At this time, the control of diabetes mellitus was poor and cholesterol level was increased. A subsequent lacunar infarct occurred 17 months after the second study, with a further increase in the extent of WML (score $=14$ ) (bottom row). 
Table 5 Multiple linear regression analysis with the increase of WML score as the dependent variable

\begin{tabular}{lllll}
\hline Variable & Coefficient & $\begin{array}{l}\text { Standard } \\
\text { error }\end{array}$ & + Value & p Value \\
\hline Moderate hypertension* (no=0, yes=1) & 0.53 & 0.16 & 3.28 & 0.0016 \\
Uncontrolled diabetes mellitus* (no=0, yes=1) & 0.39 & 0.13 & 2.99 & 0.0038 \\
Current smoking* (no=0, yes=1) & 0.33 & 0.16 & 2.95 & 0.04 \\
\hline
\end{tabular}

*During follow up.

This analysis is based on only those 79 patients with mild or no WML (white matter high intensity lesions on

T2 weighted magnetic resonance images).

severe WML (scores of 10, 14, 15, and 16) or in one patient with subsequent brain stem infarction. Among patients without stroke during follow up, only two patients without a history of stroke also showed asymptomatic progression of WML; at the start of follow up, WML scores were 0 to 1 and 2 to 3 , respectively, in these patients. In 79 patients with follow up MRI scans and no or mild WML, stepwise regression analysis showed that three variables during follow up (the presence of moderate hypertension, uncontrolled diabetes mellitus, and smoking) accounted for a significant proportion of the variances of the increased value of WML score (adjusted $\left.R^{2}=0.33 ; \mathrm{p}<0.0001\right)$; these three variables were independently correlated with the increased value of WML score (table $5)$. The other variables, including WML scores at baseline, did not contribute significantly to the magnitude of the $R^{2}$ value. Regression of the WML (a decrease in WML scores) was not found in any patient.

The follow up time of all the 80 patients with no or mild WML was 52 (19) months. When one patient with early occurrence of subsequent stroke and no follow up MRI scans was categorised as a patient without an increase in WML score, the incidence of stroke in patients with an increase in WML score and in those without was two of four and one of 76 patients, respectively. The risk of stroke in patients with an increase in WML score was significantly greater than in those without (log-rank test, $\mathrm{p}<0.0001)$.

\section{DISCUSSION}

Our study shows that after scrutinising the risk for atherothrombotic or embolic stroke, the extent of WML is a predictor of risk for subsequent stroke from arteriolosclerosis. Severe WML at baseline was an independent predictor of subsequent stroke (lacunar infarcts or haemorrhages) even after controlling for the risk factor status during follow up. Furthermore, progression of WML during follow up was associated with subsequent stroke in patients with initially mild WML. Serial evaluation of the extent of WML on MRI may be used to identify patients who are at risk of subsequent strokes.

Severe WML at baseline may be associated with a high risk of subsequent stroke through diffuse brain arteriolosclerosis ${ }^{4}$ - a risk factor for stroke that may be difficult to eliminate. Supporting this interpretation was the fact that all subsequent symptomatic strokes included lacunar infarcts and putaminal or thalamic haemorrhages, which are related to arteriolosclerosis. Correlative analysis of baseline data showed that age, the presence of hypertension, the presence of diabetes mellitus, and current smoking were independently correlated with the WML score, as found in previous studies. ${ }^{21}$ Hypertension and multiple lacunar infarcts (mostly in the basal ganglia) were interchangeable in the model, because the presence of multiple lacunar infarcts was associated with hypertension..$^{22}$ In our patient sample, the extent of WML may reflect the total effect of these risk factors on brain arterioles at entry, leading to a closer association with subsequent strokes than the presence of any one of these risk factors at entry that were potentially modifiable during follow up.
In stroke patients with medical management, the degree of control of vascular risk factors, but not their presence, may be an important determinant of stroke. Thus the strength and independence of the predictive value of WML for stroke may become clearer when risk factor status during follow up is controlled for using multivariable analysis. In our sample, the presence of moderate hypertension, uncontrolled diabetes mellitus, and smoking during follow up were associated with subsequent stroke in single variable analysis. For the prevention of stroke, these risk factors should have been more strictly controlled. However, the association between the extent of WML and the risk of subsequent stroke was independent of the risk factor status during follow up. This suggests that patients with severe WML have a high risk of subsequent stroke, even if vascular risk factors are strictly controlled. In addition, the use of antiplatelet agents did not affect the risk of stroke significantly. Thus we must seek special treatment strategies for preventing subsequent stroke in patients with severe WML and a low risk of atherothrombotic or embolic stroke. ${ }^{23-25}$

The correlation between the extent of WML and the risk of subsequent stroke is supported by the finding that progression of WML during follow up was associated with subsequent stroke in patients with initially no or mild WML. Asymptomatic progression of WML occurred in two patients with initially mild WML before the recurrence of symptomatic stroke-at the start of follow up their WML scores were 8 to 11 and 4 to 5 , repectively. Among patients without stroke occurrence during follow up, two without a history of stroke also showed asymptomatic progression of WML. However, their initial and follow up WML scores were low ( 0 to 1 and 2 to 3 ), suggesting that these patients were still at relatively low risk of stroke.

In our patients without severe WML, risk factor status during follow up was associated with progression of WML. Some conditions-including aggravation of vascular risk factors and progression of atherosclerosis or arteriosclerosis-might cause an increase in WML over time. A few population based studies on the rate of WML progression have shown asymptomatic increases in the number or extent of WML in relation to hypertension. ${ }^{13}{ }^{14}$ Asymptomatic progression of WML during follow up may indicate an increased risk of symptomatic stroke, which warrants a change of treatment, including more strict control of risk factors. Further studies are required to determine whether the identification of patients with asymptomatic deterioration of WML by serial MRI examinations may have clinical significance in preventing subsequent symptomatic stroke in those with initially mild WML. In this study, we evaluated WML on a thick MRI slice using a rather simple scale. The use of a thin slice technique and the application of semiautomated segmentation techniques which might give normalised volumes of WML or automated approaches to lesion counting could provide higher sensitivity for detecting deterioration of WML.

The predictive value of WML for stroke may be most pronounced in patients with lacunar infarction. However, it is controversial whether the extent of WML is an independent predictor of stroke in patients with lacunar infarcts. ${ }^{6912}$ One follow up CT study showed an association between the presence 
of extensive leucoaraiosis and a high risk of recurrent stroke, but only by single variable analysis. ${ }^{9}$ This association has not been supported by other studies. ${ }^{6}{ }^{12}$ The inconsistency among the previous studies may originate from possible differences in risk factor status among the patients studied. In patients with lacunar infarction, the coexistence of significant stenosis of major cerebral arteries or atrial fibrillation would increase the risk of atherothrombotic or embolic infarction. Many patients with lacunar infarction may develop recurrent non-lacunar type stroke, ${ }^{26}$ as shown in the two studies which found no association between WML or leucoaraiosis and stroke. ${ }^{6}{ }^{12}$ No patients in our study had significant stenosis of the cervical or intracranial arteries, atrial fibrillation, or ischaemic heart disease with a source of emboli, resulting in a low risk of concomitant atherothrombotic or embolic infarction. The selected nature of our patients may have resulted in the significant association of the extent of WML with subsequent stroke from arteriolosclerosis, as well as the lower recurrence rate in patients with lacunar infarction in this study compared with previous studies..$^{69^{12}}$ The predictive value for stroke of WML, as an indicator of arteriolosclerosis, becomes clear when the risk associated with WML is calculated on the basis of the occurrence of arteriolosclerotic strokes, including lacunar infarction and haemorrhages, in selected patients at low risk for atherothrombotic or embolic strokes.

The strength of our study is the careful follow up, with characterisation of the control of risk factors and serial MRI scans. However, our study is limited by the selected nature of the population, and a community based population study would have been ideal. Our patients included those with symptomatic lacunar infarcts and also stroke-free, neurologically normal patients with headache or dizziness. We did not consider the use of a control group with headaches or dizziness-as opposed to healthy, community volunteers-to be a major problem, because the control subjects were otherwise neurologically normal and had no evidence of significant pathology other than WML on MRI scanning. No report has shown any difference between the nature of WML in patients with and without lacunar infarction. Thus the nature of WML in the two patient populations in this study is probably the same as in healthy volunteers, although we cannot completely exclude the possibility that WML in patients with tensiontype headache might differ from those in patients with lacunar infarcts. In addition, the distribution of vascular risk factors was not different between the two populations, except for sex (data not shown). As the association between WML and subsequent stroke based on arteriolosclerosis was independent of the presence of lacunar infarction, this association may apply to this mixed population.

Although it is unclear whether the current findings apply to other populations, a population based study of normal adults without history of cerebrovascular disease also suggested that the presence of WML on T2 weighted MR images was an independent risk factor for subsequent subcortical stroke. ${ }^{7}$ Our study suggests that, after examining the risks for atherothrombotic or embolic stroke, patients without non-lacunar infarction but with a high WML score (9-16) should be managed as having a high risk of subsequent stroke from arteriolosclerosis (hazard ratio more than 14.4 compared with patients without WML). This scrutiny is reasonable in clinical neurological practice because the management of stroke patients is dependent on the subtypes of stroke. In this study, we confined our investigations to echocardiography to evaluate ischaemic heart disease and an ECG to exclude atrial fibrillation. More detailed investigation for sources of cardioembolism may be needed for the appropriate management of these patients

\section{Conclusions}

After scrutinising the risk for atherothrombotic or embolic stroke, severe WML at baseline was found to be an independent predictor of risk for subsequent stroke from arterioloscle- rosis even after controlling for risk factor status during follow up. Progression of WML during follow up is associated with subsequent stroke in patients with initially mild WML. Serial evaluation of the extent of WML on magnetic resonance imaging may be used to identify patients who are at risk of subsequent stroke.

\section{Authors' affiliations}

H Yamauchi, Research Institute, Shiga Medical Centre, Moriyama, Japan

H Fukuda, Department of Neurology, Saiseikai Noe Hospital, Osaka, Japan

C Oyanagi, Department of Neurology, Faculty of Medicine, Kyoto

University, Kyoto, Japan

\section{REFERENCES}

1 Pantoni L, Garcia JH. The significance of cerebral white matter abnormalities 100 years after Binswanger's report. A review. Stroke 1995;26:1293-301.

2 Longstreth WT, Manolio TA, Arnold A, et al. Clinical correlates of white matter findings on cranial magnetic resonance imaging of 3301 elderly people. The Cardiovascular Health Study. Stroke 1996;27:1274- 82.

3 Fazekas F, Kleinert R, Offenbacher H, et al. Pathologic correlates of incidental MRI white matter signal hyperintensities. Neurology 1993;43:1683-9.

4 van Swieten JC, van den $\mathrm{Hout} \mathrm{JH}$, van Ketel BA, et al. Periventricular lesions in the white matter on magnetic resonance imaging in the elderly. A morphometric correlation with arteriolosclerosis and dilated perivascular spaces. Brain 1991;114:761-74.

5 Kapeller P, Schmidt R. Concepts on the prognostic significance of white matter changes. I Neural Transm Suppl 1998;53:69-78.

6 Clavier I, Hommel M, Besson G, et al. Long-term prognosis of symptomatic lacunar infarcts. A hospital-based study. Stroke 1994;25:2005-9.

7 Kobayashi S, Okada K, Koide H, et al. Subcortical silent brain infarction as a risk factor for clinical stroke. Stroke 1997:28:1932-9.

8 van Swieten JC, Kappelle L, Algra A, et al. Hypodensity of the cerebral white matter in patients with transient ischemic attack or minor stroke: influence on the rate of subsequent stroke. Dutch TIA Trial Study Group. Ann Neurol 1992;32:177-83.

9 Miyao S, Takano A, Teramoto J, et al. Leukoaraiosis in relation to prognosis for patients with lacunar infarction. Stroke 1992;23:1434-8.

10 Inzitari D, Di Carlo A, Mascalchi M, et al. The cardiovascular outcome of patients with motor impairment and extensive leukoaraiosis. Arch Neurol 1995;52:687-91.

11 Briley DP, Haroon S, Sergent SM, et al. Does leukoaraiosis predict morbidity and mortality? Neurology 2000;54:90-4.

12 Salgado AV, Ferro JM, Gouveia-Oliveira A. Long-term prognosis of first-ever lacunar strokes. A hospital-based study. Stroke 1996;27:661-6.

13 Veldink JH, Scheltens P, Jonker C, et al. Progression of cerebral white matter hyperintensities on MRI is related to diastolic blood pressure. Neurology 1998;51:319-20.

14 Schmidt R, Fazekas F, Kapeller P, et al. MRI white matter hyperintensities. Three year follow-up of the Austrian Stroke Prevention Study. Neurology 1999;53:132-9.

15 Fisher CM. Lacunar strokes and infarcts: a review. Neurology 1982;32:871-6.

16 Guidelines Subcommittee. 1993 guidelines for the management of mild hypertension. Memorandum from a World Health Organisation/International Society of Hypertension meeting. J Hypertens 1993:11:905-18.

17 Guidelines Subcommittee. 1999 World Health Organization/ International Society of Hypertension Guidelines for the management of hypertension. J Hypertens 1999;17:151-83.

18 van Swieten JC, Hiidra A, Koudstaal PJ, et al. Grading white matter lesions on CT and MRI: a simple scale. J Neurol Neurosurg Psychiatry 1990;53:1080-3

19 Kaplan EL, Meier P. Nonparametric estimation from incomplete observations. J Am Stat Assoc 1958;53:457-81.

20 Cox DR. Regression models and life tables. J R Stat Soc Ser B 1972;34:187-220.

21 Fukuda H, Kitani M. Cigarette smoking is correlated with the periventricular hyperintensity grade of brain magnetic resonance imaging. Stroke 1996;27:645-9.

22 Boiten J, Lodder J, Kessels F. Two clinically distinct lacunar infarct entities? A hypothesis. Stroke 1993;24:652-6.

23 Caplan LR. Binswanger's disease - revisited. Neurology 1995;45:626-33

24 van Zagten $M$, Boiten J, Kessels F, et al. Significant progression of white matter lesions and small deep (lacunar) infarcts in patients with stroke. Arch Neurol 1996;53:650-5.

25 Akiguchi I, Tomimoto $H$, Kinoshita $M$, et al. Effects of antithrombin on Binswanger's disease with antiphospholipid antibody syndrome. Neurology 1999:52:398-401.

26 Yamamoto H, Bogousslavsky J. Mechanisms of second and further strokes. J Neurol Neurosurg Psychiatry 1998;64:771-6. 